\title{
Taquicardia postural ortostática en 15 pacientes: disautonomía compleja
}

\author{
PEDRO JIMÉNEZ-COHL ${ }^{1}$, NICHOLAS EARLE M. ${ }^{\text {a }}$, \\ BELTRÁN GONZÁLEZ R. ${ }^{2}$, ELFRIDE THIECK J. ${ }^{1, b}$
}

\section{Postural orthostatic tachycardia syndrome (POTS). Report of 15 cases}

Background: Patients with postural orthostatic tachycardia syndrome (POTS) report dizziness, lightheadedness, weakness, blurred vision, and fatigue upon standing. The diagnosis of the syndrome is made when an orthostatic intolerance and tachycardia appear in the standing position. Aim: To report 15 patients with POTS. Material and Methods: Review of Tilt test reports in a period of 15 years. Those reports in which orthostatic postural tachycardia and symptoms compatible with POTS appeared, were selected for analysis. Results: We identified 15 patients $(3.1 \%$ of all positive Tilt test reports) with compatible signs and symptoms. There was a lag of 8-10 years between the onset of symptoms and the time of diagnosis. Most patients complained of orthostatic intolerance, dizziness and frequent fainting. Orthostatic tachycardia and symptoms occurred on average after 2.9 and 6.1 minutes, respectively, of staying in the standing position. These patients had a high frequency of family history of syncope or presyncope (66\% frequency) and hypermobility syndrome (53\% prevalence). Only 33\% of the patients reported relief of their symptoms after being treated (most of them with fludrocortisone). Most patients that reported little or no relief, did not use medications or were treated for a short period. Conclusions: POTS syndrome is uncommon but disturbs quality of life of those who suffer it. Its association with hypermobility syndromes must be investigated.

(Rev Med Chile 2012; 140: 145-152).

Key words: Hypermobility syndrome; Postural orthostatic tachycardia syndrome; Tilt-table test.

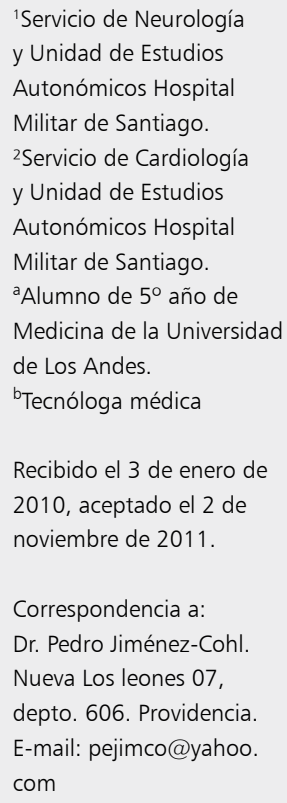

E 1 síndrome de taquicardia postural ortostática (POTS, como es conocido por su sigla en inglés) es un tipo de disautonomía clínicamente caracterizada por intolerancia ortostática y taquicardia dentro de los primeros 10 minutos de la estadía de pie, con una frecuencia cardiaca en pie que supera en 30 pulsaciones a la frecuencia basal (acostado) o que sobrepasa los 120 latidos en total. La intolerancia ortostática se define como la aparición de síntomas de hipoperfusión cerebral al estar de pie, que se alivian al acostarse ${ }^{1-7}$. Muchas de las actividades de la vida diaria pueden estar interferidas por las molestias ${ }^{8,9}$.

Los pacientes clásicamente no consultan mé- dico por mucho tiempo o son erróneamente diagnosticados como depresión, estrés, fatiga crónica o trastorno de pánico ${ }^{7,10}$, se quejan de fatiga extrema, intolerancia al ejercicio, mareos, disminución de la concentración y síncopes ${ }^{1-8}$.

En el Laboratorio de Estudios Autonómicos de nuestro Hospital, hemos estudiado 15 casos en los que la taquicardia ortostática ocurre dentro de los primeros 10 minutos al ponerse de pie, cumpliendo por lo tanto la definición ${ }^{1-3}$.

Estudiamos en forma retrospectiva a los pacientes en cuanto a los síntomas de consulta, los hallazgos hemodinámicos en el Tilt Test (TT), la respuesta al tratamiento, evolución y pronóstico. 


\section{Material y Métodos}

Revisamos desde marzo de 1996 hasta mayo 2011, 630 exámenes de Tilt Test del Laboratorio de Estudios Autonómicos del Departamento de Neurología del Hospital Militar de Santiago. De éstos, 473 (75\%) demostraron ser positivos para disautonomía. Encontramos 15 casos (3,1\% de las pruebas positivas) en el que los pacientes presentaban taquicardia ortostática postural y síntomas en el Tilt Test concordantes con la definición de POTS $^{1-3}$.

En la Tabla 1 se describen las características demográficas de los 15 pacientes al momento del diagnóstico. A 15 controles sanos, pareados por sexo y edad con los pacientes; en ambos grupos se aplicó el mismo protocolo de TT.

\section{Protocolo utilizado}

Siempre el TT se realizó en la mañana entre las 8 y las 10 horas, los pacientes y los controles fueron atendidos en ayunas.

Participaron un neurólogo, un cardiólogo y un tecnólogo médico, además se contaba con la colaboración del personal de la cardiología en la prestación de apoyo instrumental, acceso venoso, monitorización cardiaca continua y el manejo de la infusión e.v. de isoprotenerol.

En pacientes y controles se efectuó igual protocolo:

a) La prueba de respiración profunda (también llamado índice de RR).

b) La maniobra de Valsalva, y

c) Examen de Tilt Test (TT).

Para asegurar que los pacientes no tuviesen una hipoglicemia, en todos los casos un hemoglucotest fue practicado antes de iniciar el examen.

\section{El Tilt Test se divide en fases}

I) Paciente en reposo en posición horizontal durante 10 minutos, para obtener los parámetros basales de presión arterial basal (PA) y de frecuencia cardiaca (FC)

II) Tilt Test pasivo (sin activador), paciente de pie durante 45 minutos como máximo, con una inclinación de $80^{\circ}$. Este grado de inclinación se varió a $70^{\circ}$ después de este trabajo.

III) Tilt Test activado. Se recuesta al paciente horizontalmente durante 5 minutos. Una infusión de isoprotenerol (activador) se administra a través de una vía venosa. $1 \mathrm{ml}(0,2 \mathrm{mg}=200 \mu \mathrm{g})$ disueltos en $200 \mathrm{ml}$. de suero en dextrosa al 5\%, a una velocidad de $180 \mathrm{ml}$ por hora $(3 \mu \mathrm{g} / \mathrm{min})$.

IV) El paciente es ubicado de pie en la inclinación de $80^{\circ}$, por un máximo de 10 minutos, mientras que pasa la infusión de isoprotenerol e.v. $(3 \mu \mathrm{g} / \mathrm{min})$.

V) La prueba termina en cualquier momento si el paciente tiene lo que se puede llamar un "TT positivo"; esto es síncope (pérdida de conciencia) o presíncope (signos que anuncian que el síncope es inminente) asociado con hipotensión o hipotensión más bradicardia, o si se producen molestias intolerables para el paciente.

Si no hay síntomas se finaliza debido al término del protocolo.

El equipamiento técnico del TT se compone de: Monitor digital (Ohmeda 2300 Finapres BP Monitor USA), el brazalete se coloca en el dedo índice o medio para medir la PA y la FC en forma continua; camilla para Tilt Test (Magnetic Manumed USA) alimentada por electricidad con una velocidad de inclinación que va desde la posición

Tabla 1. Sexo y edad al momento del inicio de los síntomas y del diagnóstico

\begin{tabular}{|lllcc|}
\hline & Femenino & Masculino & $\begin{array}{c}\text { Edad al inicio de los } \\
\text { síntomas (años) }\end{array}$ & $\begin{array}{c}\text { Edad al momento del } \\
\text { diagnóstico (años) }\end{array}$ \\
Pacientes & 10 casos & 5 casos & 14,07 & 22,6 \\
N: 15 casos & & & & \\
Edad: 22,6 años & & & \\
Rango: $9-43$ años & & & \\
Controles & 10 voluntarias & 5 voluntarios & & \\
N: 15 & & & \\
Edad: 25,6 años & & & \\
Rango: $10-50$ años & & & \\
\hline
\end{tabular}


POTS en 15 pacientes - P. Jiménez-Cohl et al

acostada $\left(180^{\circ}\right)$ hasta de pie a $80^{\circ}$ en 27 segundos (aproximadamente $3^{\circ}$ por segundo).

Monitor de electrocardiograma (Quinton Q4500 USA) para vigilar la frecuencia y la forma del latido cardiaco. Bomba de infusión para isoprotenerol: LifeCare-XLUSA.

Los parámetros hemodinámicos y los síntomas son registrados. Todos nuestros estudios han sido aprobados por el comité de ética del Hospital, y se han realizado de conformidad con las normas éticas establecidas en la Declaración de Helsinki 1964. Los pacientes y los controles dieron su consentimiento informado antes de su inclusión en el estudio.

\section{Resultados}

Los síntomas por los cuales los pacientes consultan aparecen en la Tabla 2.

\section{Historia familiar de síncope o presíncope en familiares de primer grado}

Pacientes: 10/15 con antecedentes familiares $=66 \%$.

Controles: 0/15 con antecedentes familiares de síncope o presíncope.

Hiperlaxitud ligamentosa: Es un hallazgo descrito en pacientes con POTS o con otra clase de disautonomías ${ }^{11-13}$. De hecho, según los "Criterios de Brighton" 14 presentaban hiperlaxitud ligamentosa 8 de 15 de nuestros casos (53\%) y también sus familiares cercanos. Sólo dos controles presentaban hiperlaxitud (13\%).

\section{Hallazgos en el Tilt Test}

En la Tabla 3 se describen los parámetros de FC y PA en reposo, en posición de TT pasivo y durante el TT activado, para pacientes y controles. la etapa de TT activado, sólo llegaron 6 pacientes, ya que los otros 9 tuvieron un TT positivo en la fase de TT pasivo.

La FC en el TT pasivo (Tabla 3) alcanzó como rango 100-132 latidos en los pacientes, mientras que en los controles no pasó de 95 latidos. Así, la frecuencia cardiaca media en esa fase (Tabla 3 y Figura 1), marcó una diferencia estadísticamente significativa entre pacientes y controles (111 vs $87 \mathrm{p} \leq 0,01)$.

Por el contrario, en la PA sistólica y diastólica no se encontraron diferencias significativas entre pacientes y controles ni en reposo ni durante las 2 fases del Tilt Test (p: no significativo).

La taquicardia ortostática en los pacientes se alcanzó en promedio a los 2,9 minutos (Tabla 4). En $9 / 15$ casos $(60 \%)$, este valor se alcanzó en el minuto 1 de estar en la posición vertical.

Tabla 2. Síntomas de consulta y su frecuencia en nuestra muestra

\begin{tabular}{|lcc|}
\hline & \multicolumn{2}{c|}{ Pacientes } \\
& (n) & (\%) \\
\hline Intolerancia ortostática & $7 / 15$ & 47 \\
Síncopes frecuentes & $7 / 15$ & 47 \\
Lipotimias frecuentes & $5 / 15$ & 33 \\
Síncopes ocasionales & $4 / 15$ & 27 \\
Intolerancia al esfuerzo & $3 / 15$ & 20 \\
\hline Síncope después de esfuerzos & $1 / 15$ & 7 \\
\hline
\end{tabular}

Tabla 3.

\begin{tabular}{|c|c|c|}
\hline \multicolumn{3}{|c|}{$\begin{array}{l}\text { Parámetros hemodinámicos en el Tilt Test } \\
\text { en reposo por } 10 \text { minutos }\end{array}$} \\
\hline & Pacientes n: 15 & Controles n: 15 \\
\hline PA promedio & $103 / 62$ & $110 / 60$ \\
\hline PA Rango & $85 / 58-118 / 75$ & $90 / 62-120 / 65$ \\
\hline FC promedio & 78 & 80 \\
\hline F C Rango & $65-102$ & $67-90$ \\
\hline \multicolumn{3}{|c|}{$\begin{array}{l}\text { Parámetros hemodinámicos en el Tilt Test } \\
\text { pasivo en pie ( } 45 \text { minutos) a } 80^{\circ} \text { de inclinación }\end{array}$} \\
\hline & $\begin{array}{l}\text { Pacientes n: } 15 \\
\text { (Sin síntomas) }\end{array}$ & Controles n: 15 \\
\hline PA promedio & $118 / 72$ & $120 / 75$ \\
\hline PA Rango & $105 / 56-125 / 88$ & $110 / 60-130 / 90$ \\
\hline FC promedio & 111 & 87 \\
\hline F C Rango & $100-132$ & $80-95$ \\
\hline \multicolumn{3}{|c|}{$\begin{array}{l}\text { Parámetros hemodinámicos en el TT activado } \\
\text { (isoprotenerol) en pie a } 80^{\circ} \text { de inclinación }\end{array}$} \\
\hline & $\begin{array}{l}\text { Pacientes n: } 6 \\
\text { Pre síntomas }\end{array}$ & Controles n: 15 \\
\hline PA promedio & $115 / 70$ & $118 / 73$ \\
\hline PA Rango & $100 / 55-125 / 83$ & $110 / 60-128 / 85$ \\
\hline FC promedio & 130 & 127 \\
\hline F C Rango & $115-145$ & $117-137$ \\
\hline
\end{tabular}


Tabla 4. Tiempo que se demora de pie en llegar a una FC, mayor o igual en $\mathbf{3 0}$ latidos con respecto a la basal (acostado)

\begin{tabular}{|ccc|}
\hline $\begin{array}{c}\text { Paciente } \\
\mathbf{N}^{\circ}\end{array}$ & $\begin{array}{c}\text { Tiempo } \\
\text { (minutos) }\end{array}$ & $\begin{array}{c}\text { FC } \mathbf{3 0} \text { latidos } \\
\text { > basal }\end{array}$ \\
1 & 1 & 105 \\
2 & 3 & 107 \\
3 & 1 & 112 \\
4 & 1 & 100 \\
\hline 5 & 5 & 110 \\
6 & 1 & 117 \\
\hline 7 & 10 & 114 \\
\hline 8 & 3 & 100 \\
9 & 3 & 113 \\
10 & 1 & 103 \\
11 & 1 & 132 \\
\hline 12 & 1 & 118 \\
13 & 1 & 115 \\
\hline 14 & 10 & 103 \\
\hline 15 & 1 & 120 \\
Promedio & 2,9 minutos & 111 \\
Rango & $1-10$ minutos & $100-132$ \\
D. S. & 3,1 & 8,7 \\
\hline
\end{tabular}

Los síntomas ortostáticos en el Tilt Test se describen en la Tabla 5.

En promedio, los síntomas ortostáticos se presentaron a los 6,1 minutos (rango de 1 a $10 \mathrm{~min}$ ).Como "presíncope", se consideró que el paciente tenía una combinación de síntomas como: palidez, visión borrosa, sudoración y mareos, situación que permite saber que el síncope es inminente, asociado a una baja significativa de la PA en la posición de pie. Los parámetros hemodinámicos previos a la aparición de síntomas en el TT pasivo, con el paciente de pie por 45 minutos, fueron PA de 109/71 mmHg y FC de 112 latidos por minuto.

Como evento final en el TT, se produjo un presíncope (lipotimia) en 14 de 15 casos y severa molestia por intolerancia ortostática en uno de los 15. En la Tabla 6 podemos ver el tipo de evento final en el examen.
Tabla 5. Sintomatología ortostática en Tilt Test

\begin{tabular}{|clc|}
\hline $\begin{array}{c}\text { Paciente } \\
\mathbf{N}^{\circ}\end{array}$ & $\begin{array}{l}\text { Sintomatología } \\
\text { al estar de pie }\end{array}$ & $\begin{array}{c}\text { Tiempo para } \\
\text { legar a los } \\
\text { síntomas }\end{array}$ \\
1 & Mareos + intolerancia ortostática & 1 \\
2 & Intolerancia ortostática & 3 \\
3 & Intolerancia ortostática & 8 \\
4 & Intolerancia ortostática & 10 \\
5 & Intolerancia ortostática & 5 \\
6 & Intolerancia ortostática + mareos & 5 \\
7 & Mareos + intolerancia ortostática & 10 \\
8 & Mareos + intolerancia ortostática & 8 \\
9 & Mareos + intolerancia ortostática & 6 \\
10 & Int. ortostática + presíncope & 10 \\
11 & Intolerancia ortostática & 10 \\
12 & Mareos & 5 \\
13 & Mareos & 1 \\
14 & Intolerancia ortostática & 5 \\
15 & Intolerancia ortostática & 5 \\
Promedio & & 6,1 minutos \\
& & rango: $1-10)$ \\
\hline
\end{tabular}

Tabla 6. Evento final en el Tilt Test

\begin{tabular}{|llc|}
\hline Tipo de evento / Pacientes & n & \% \\
\hline Presíncope mixto & 6 & 40 \\
\hline Presíncope vaso depresor & 8 & 53 \\
\hline Intolerancia ortostática severa & 1 & 7 \\
\hline
\end{tabular}

La intolerancia ortostática la definimos como "un notable cansancio al estar de pie con intensos deseos de sentarse o acostarse".

La respuesta al tratamiento en los pacientes entrevistados en mayo 2011, aparece en la Tabla 7.

Para evaluar la evolución de éstos, clasificamos sus síntomas en la de la siguiente manera: Síncope, lipotimia o intolerancia ortostática ocasional: hasta un episodio por mes o menos. Síncope, lipotimia o intolerancia ortostática frecuente: más de un episodio por mes. Síncope, lipotimia o intolerancia ortostática permanente si se tiene el síntoma cada vez que se pone de pie. 
Tabla 7. Respuesta a tratamiento en los pacientes examinados en mayo 2011

\begin{tabular}{|c|c|c|c|c|c|}
\hline $\begin{array}{l}\text { Paciente } \\
\text { No }^{\circ}\end{array}$ & $\begin{array}{c}\text { Evolución } \\
\text { post- } \\
\text { diagnóstico } \\
\text { (años) }\end{array}$ & $\begin{array}{l}\text { Tratamiento } \\
\text { usado y por } \\
\text { cuánto tiempo }\end{array}$ & Tratamiento actual & $\begin{array}{c}\text { Tiempo } \\
\text { para } \downarrow^{*} \\
\text { Síntomas }\end{array}$ & $\begin{array}{l}\text { Síntomas } \\
\text { actuales** }\end{array}$ \\
\hline 1 & 12 & $\begin{array}{l}\text { Florinef } \\
12 \text { meses }\end{array}$ & $\begin{array}{l}\text { Florinef ocasional } 11 \\
\text { años }\end{array}$ & 2 meses & Alivio significativo \\
\hline 2 & 2 & Florinef 2 años & Florinef permanente & 1 mes & Alivio significativo \\
\hline 3 & 15 & $\begin{array}{l}\text { Florinef } \\
3 \text { años }\end{array}$ & $\begin{array}{l}\text { Florinef ocasional } 12 \\
\text { años }\end{array}$ & 6 meses & Alivio total \\
\hline 4 & 8 & No & Nada por 8 años & No tiene alivio & $\begin{array}{l}\text { Intolerancia ortostática } \\
\text { permanente }\end{array}$ \\
\hline 5 & 15 & $\begin{array}{l}\text { Florinef } \\
15 \text { años }\end{array}$ & Florinef permanente & $\begin{array}{l}1 \text { año } \\
\text { Escaso alivio }\end{array}$ & $\begin{array}{l}\text { Intolerancia ortostática } \\
\text { frecuente }\end{array}$ \\
\hline 6 & 7 & $\begin{array}{l}\text { Atenolol } \\
6 \text { meses al principio }\end{array}$ & $\begin{array}{l}\text { Midodrina } \\
\text { Por } 1 \text { año actualmente }\end{array}$ & Escaso alivio & $\begin{array}{l}\text { Intolerancia ortostática } \\
\text { frecuente }\end{array}$ \\
\hline 7 & 7 & $\begin{array}{l}\text { Midodrina } \\
1 \text { año }\end{array}$ & Nada por 6 años & $\begin{array}{l}2 \text { meses } \\
\text { Escaso alivio }\end{array}$ & $\begin{array}{l}\text { Intolerancia ortostática } \\
\text { ocasional }\end{array}$ \\
\hline 8 & 7,5 & $\begin{array}{l}\text { Propranolol } \\
6 \text { meses }\end{array}$ & Nada por 7 años & Escaso alivio & $\begin{array}{l}\text { Intolerancia ortostática } \\
\text { frecuente }\end{array}$ \\
\hline 9 & 3 & $\begin{array}{l}\text { Midodrina por } 2 \text { años } \\
\text { al principio }\end{array}$ & Florinef por 1 año & 2 años & Alivio significativo \\
\hline 10 & 7 & No & Nada por 7 años & Sin alivio & $\begin{array}{l}\text { Intolerancia ortostática } \\
\text { frecuente }\end{array}$ \\
\hline 11 & 7 & $\begin{array}{l}\text { Midodrina \& Florinef } \\
\text { los } 3 \text { últimos años }\end{array}$ & $\begin{array}{l}\text { Midodrina \& Florinef } \\
\text { permanente }\end{array}$ & 2 años & Alivio significativo \\
\hline 12 & 5,5 & $\begin{array}{l}\text { Midodrina ocasional- } \\
\text { mente por } 1 \text { año }\end{array}$ & Nada por 4,5 años & Sin alivio & $\begin{array}{l}\text { Presíncopes \& Int. or- } \\
\text { tostática frecuentes }\end{array}$ \\
\hline 13 & 5,5 & $\begin{array}{l}\text { Midodrina \& Florinef } \\
\text { por } 3 \text { años }\end{array}$ & $\begin{array}{l}\text { Midodrina \& Florinef } \\
\text { por } 3 \text { años }\end{array}$ & Escaso alivio & $\begin{array}{l}\text { Intolerancia ortostática } \\
\text { y al esfuerzo }\end{array}$ \\
\hline 14 & 7 & Midodrina 4 meses & Nada por 6,5 años & Sin alivio & Int. ortostática frecuente \\
\hline 15 & 4,5 & Midodrina por 2 años & Nada por 2,5 años & Sin alivio & Int. ortostática frecuente \\
\hline
\end{tabular}

*Tiempo para $\downarrow$ síntomas: 1) Sin alivio implica nada de mejoría. 2) Alivio escaso: Es que las molestias de intolerancia ortostática o presíncope el paciente las percibe levemente mejor pero no logran bajar a la categoría de síntoma ocasional. 3) Síntoma ocasional: hasta un episodio por mes. 4) Alivio significativo se considera un episodio sintomático cada 3 meses o menos. **Síntomas actuales: Intolerancia ortostática o presíncope frecuente: Más de un episodio por mes. Intolerancia ortostática o presíncope ocasional: Hasta un episodio por mes o menos. Alivio significativo se considera un episodio sintomático cada 3 meses o menos.

Intolerancia al esfuerzo ocasional: el paciente puede caminar una cuadra, sin síntomas. Intolerancia al esfuerzo frecuente: el paciente no puede caminar una cuadra, sin tener síntomas.

\section{Discusión}

POTS es una entidad clínica en que los pacientes son diagnosticados mediante el examen de $\mathrm{TT}^{15-17}$, cuando la taquicardia y los síntomas ortostáticos, se producen dentro de los 10 minutos de la estadía de pie.

En nuestro estudio encontramos que es un cuadro de poca frecuencia dentro del gran universo de las disautonomías; representando sólo el 3,1\% del total de exámenes de Tilt Test positivos.

Nuestros datos muestran que la aparición de los síntomas es de aproximadamente 8-10 años 


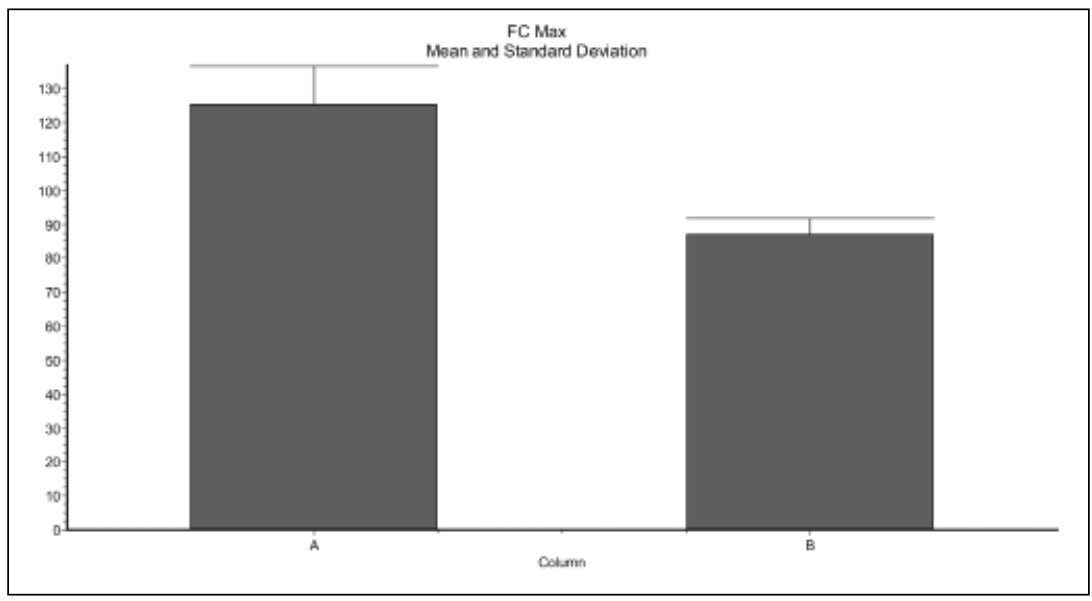

Figura 1. FC Máxima en pie (Tilt Test pasivo, sin activador). A: Casos. B: Controles; p: $<0,01$ Muy significativo. antes que se haga el diagnóstico, lo que indica que este diagnóstico es tardío y complejo. Es muy frecuente que los pacientes pasen años con diagnósticos como depresión, ansiedad o trastorno de pánico, de hecho todos en algún momento de su evolución eran medicados con ansiolíticos o antidepresivos. Creemos que esto ocurre por la poca difusión que tiene este cuadro en la comunidad médica y en el público general, donde síntomas como intolerancia ortostática o síncopes son casi siempre atribuidos a estrés emocional.

En cuanto a los síntomas de consulta la "intolerancia ortostática" y los "síncopes frecuentes" son la primera causa de consulta ( $47 \%$ de frecuencia para cada uno). La intolerancia ortostática es el síntoma más mencionado en la literatura para POTS $^{2,3,5,6-9}$, lo que coincide con nuestra observación

Fue común en nuestros pacientes encontrar una historia familiar de molestias ortostáticas 10/15 (66\%) tenían familiares de primer grado con historia de intolerancia ortostática o presíncope. Interesante observación, que nos diferencia de las publicaciones tradicionales en las que no se describe el carácter familiar ${ }^{2,3,5,9}$. Incluso los casos 3,4 y 5 corresponden a una madre y sus 2 hijos.

Es posible que se deba a la gran frecuencia en Chile del Ehlers-Danlos tipo III ${ }^{11,12}$. De hecho 8 de nuestros casos (54\%) presentaban hiperlaxitud ligamentosa ${ }^{14}$, con familiares de ler grado afectados también por hiperlaxitud pero de distinta intensidad. Años atrás se llamaba al POTS "síndrome del prolapso de la válvula mitral” ${ }^{18}$. Este prolapso mitral puede deberse a la hiperlaxitud del conectivo valvular mitral, así como también causar la hiperdistensibilidad venosa favorecedora de sintomatología ortostática ${ }^{11-13,18}$.

Otro punto importante es que los pacientes no nos dan una historia de infección viral previa a la aparición de los síntomas, un hecho totalmente diferente de otras publicaciones ${ }^{2,3,5,9}$.

El aumento de la FC es muy rápido al poner de pie al paciente. Nuestro promedio de 2,9 minutos está dentro del límite de 10 minutos sugerido por los trabajos pioneros de Phillip Low ${ }^{1-3}$.

Los síntomas ortostáticos aparecieron en el TT en promedio a los 6,1 minutos de estar de pie. Estos fueron la intolerancia ortostática $(86 \%)$, el mareo ortostático $(46 \%)$ y el presíncope $(6 \%)$.

El seguimiento de la serie (Tabla 7), va de 2 a 15 años. Se incluyen desde casos sin ningún tratamiento, hasta pacientes con 2 medicamentos asociados. Los fármacos utilizados fueron fludrocortisona, atenolol, midodrina y propranolol. Otros tratamientos mencionados en la literatura como inhibidores de la acetilcolinesterasa y sobrecarga de volumen no se utilizaron en nuestros pacientes ${ }^{19,20}$.

En cuanto al tratamiento; 13 pacientes consumieron fármacos en algún momento de su evolución, y los otros 2 no (uno de ellos por decisión propia no deseaba usar fármacos y el otro dijo que nunca fue medicado). Estos dos casos no se recuperaron nunca de sus síntomas.

El promedio de tiempo total de uso de fármacos fue de 27,3 meses. 
POTS en 15 pacientes - P. Jiménez-Cohl et al

Tabla 8. Efectividad de los medicamentos usados

\begin{tabular}{|c|c|c|}
\hline $\begin{array}{l}\text { Magnitud } \\
\text { del alivio }\end{array}$ & $\begin{array}{l}\text { Pctes } \\
\text { (n) }\end{array}$ & Medicamentos utilizados \\
\hline $\begin{array}{l}\text { Alivio } \\
\text { total }\end{array}$ & 1 & Florinef $\rightarrow$ ocasional: 1 caso \\
\hline $\begin{array}{l}\text { Alivio } \\
\text { significativo }\end{array}$ & 4 & $\begin{array}{l}\text { Florinef: } 3 \text { casos } \\
\text { Midodrina } \rightarrow \text { Florinef: } 1 \text { caso }\end{array}$ \\
\hline $\begin{array}{l}\text { Alivio } \\
\text { escaso }\end{array}$ & 5 & $\begin{array}{l}\text { Abandono de tratamiento: } 2 \text { casos } \\
\text { Midodrina ocasional: } 1 \text { caso } \\
\text { Florinef + Midodrina: } 1 \text { caso } \\
\text { Florinef ocasional: } 1 \text { caso }\end{array}$ \\
\hline $\begin{array}{l}\text { Alivio } \\
\text { ninguno }\end{array}$ & 5 & $\begin{array}{l}\text { Sin tratamiento: } 2 \text { casos } \\
\text { Abandono de tratamiento } \\
\text { (Midodrina): } 3 \text { casos }\end{array}$ \\
\hline
\end{tabular}

Vemos una importante tendencia en la muestra a abandonar los tratamientos y no controlarse con médico. Así, 6/15 (40\%) de los casos abandonaron el tratamiento, más los 2 casos (13\%) que jamás recibieron tratamiento constituyen el $53 \%$ de nuestra muestra. Esto nos hace pensar en la gran necesidad de educar a pacientes y médicos sobre los tratamientos existentes y que sí es posible aliviar las molestias.

Por lo demás como vemos el mejor medicamento para encontrar algún alivio lo constituye el Florinef solo o combinado con midodrina.

Los pacientes que tuvieron alivio total o significativo constituyen sólo el $33 \%$ y con escaso o ningún alivio el 66\%. Sólo uno (6\%) de los pacientes refirió que bajo tratamiento se encontraba totalmente aliviado de sus síntomas. Por otra parte, el $33 \%$ de los casos nunca ha encontrado alivio a sus molestias.

Los pacientes 1, 2, 3, 9 y 11 son aquellos que se pueden considerar con una mejor evolución o sea con mayor alivio bajo tratamiento, consideraban que sus síntomas ocurrían muy ocasionalmente, les pregunté si lo evaluarían como un episodio cada 3 meses o menos ( 4 veces o menos en un año)

Para ver los tratamientos según su efectividad podemos ver la Tabla 8 .

En resumen, POTS es un tipo de disautonomía no muy frecuente, pero que afecta importantemente la vida cotidiana de los que la sufren, ya que produce una intolerancia ortostática muy difícil de aliviar. Nos parece que en nuestros casos existe un fuerte factor hereditario y que también debe investigarse a futuro la relación con el síndrome de Ehlers Danlos.

Las diferentes respuestas al tratamiento en los distintos pacientes, puede representar la existencia de distintos subtipos de POTS, hecho que ya han planteado algunos autores ${ }^{21-26}$ y que debe aclararse.

Agradecimientos: Se contó con la colaboración del personal de auxiliares de enfermería del Servicio de Cardiología, quienes instalaron el monitoreo electrocardiográfico: Mónica San Martín, Carmen Vidal, Carmen Navarro y María Elena Caballero. Las enfermeras del Servicio de Cardiología, encargadas de la instalación de la vía venosa y de la infusión de isoprotenerol, a través de bomba de infusión fueron: Ana Castro, Jeannette Orellana y Leonor Acevedo. Hago un especial recuerdo de Beltrán González, gran colega y colaborador fallecido en abril 2010. Mi deber es lograr que este trabajo sea difundido.

\section{Referencias}

1. Schondorf R. Low P Idiopathic Postural Tachycardia Syndrome. Annals of neurology 1990; 28 (2) (Program \& abstracts) (P210): 271.

2. Schondorf R, Low P. Idiopathic postural orthostatic tachycardia syndrome: An attenuated form of acute pandyssautonomia. Neurology 1993; 43: 132-7.

3. Low PA, Opfer-Gehrking TL, Textor SC, Benarroch EE, Shen WK, Schondorf R, Suarez GA, Rummans TA Postural tachycardia syndrome (POTS). Neurology 1995; 45: S19-25.

4. Rosen SG, Cryer PE. Postural tachycardia syndrome: Reversal of sympathetic hyperresponsiveness and clinical improvement during sodium loading. The American Journal of medicine 1982; 72: 847-50.

5. Grubb BP. Postural Tachycardia Syndrome. Circulation 2008; 117: 2814-7.

6. Thieben MJ, Sandroni P, Sletten DM, Benrud-Larson LM, Fealey R, Vernino S, et al. Postural Orthostatic Tachycardia Syndrome: The Mayo Clinic Experience. Mayo Clinic Proceedings 2007; 82 (3): 308-13.

7. Low PA, Sandroni P, Joyner M, Shen WK. Postural Tachycardia Syndrome (POTS): Clinical Features. Medscape 2009 [Consultado el 1 de mayo 2011]. Disponible en: http://www.medscape.com/viewarticle/705183_2?src=e mailthis.

8. Benrud-Larson LM, Sandroni P, Haythornthwaite JA, Rummans TA, Low PA. Correlates of functional disa- 
bility in patients with postural tachycardia syndrome: preliminary cross-sectional findings. Health Psychol 2003; 22(6): 643-8.

9. Sandroni P, Opfer-Gehrking TL, McPhee BR, Low PA. Postural tachycardia syndrome: clinical features and follow-up study. Mayo Clin Proc 1999; 74 (11): 1106-10.

10. Masuki S, Eisenach JH, Johnson CP, Dietz NM, BenrudLarson LM, Schrage WG, et al. Excessive heart rate response to orthostatic stress in postural tachycardia syndrome is not caused by anxiety. J Appl Physiol 2006; 102 (3): 896-903. Epub 2006 Nov 16.

11. Bravo SJ. Disautonomía: Un problema médico poco conocido. Jaime Bravo Silva. 2004. Disponible en: http:// www.reumatologia-dr-bravo.cl/paramedicos/articulos/ DisBHSJD.htm. [Consultado el 1 de enero 2011].

12. Bravo SJ. Síndrome de Ehlers-Danlos tipo III, llamado también Síndrome de Hiperlaxitud Articular (SHA): Epidemiología y manifestaciones clínicas. Rev Chil reumatol 2010; 26 (2): 194-202.

13.- Rowe PC, Barron DF, Calkins H, Maumenee IH, Tong PY, Geraghty MT. Orthostatic intolerance and chronic fatigue syndrome associated with Ehlers Danlos syndrome. J Pediatr 1999; 135: 494-9.

14. Grahame R. The revised (Brighton 1998) criteria for the diagnosis of benign joint hypermobility syndrome (BJHS). J Rheumatol 2000; 27: 1777-9.

15. Kapoor WN. Using a tilt table to evaluate syncope. Am J Med Sci 1999; 317(2): 110-6.

16. Al-Shekhlee A, Lindenberg JR, Hachwi RN, Chelimsky TC. The value of autonomic testing in postural tachycardia syndrome. Clin Auton Res 2005; 15 (3): 219-22.

17. Grubb BP, Kanjwal Y, Kosinski DJ. The postural tachycardia syndrome: a concise guide to diagnosis and manage- ment. J Cardiovasc Electrophysiol 2006; 17 (1): 108-12.

18. Simpson MR. Benign Joint Hypermobility Syndrome: Evaluation, Diagnosis, and Management JAOA 2006; 106 (9): 531-6 [Consultado el 1 de mayo, 2011]. Disponible en: http://www.jaoa.org/cgi/content/full/106/9/531.

19. Raj SR, Black BK, Biaggioni I, Harris PA, Robertson D. Acetylcholinesterase inhibition improves tachycardia in postural tachycardia syndrome. Circulation 2005; 111 (21): 2734-40.

20. Jacob G, Shannon JR, Black B, Biaggoni I, MosquedaGarcía R, Robertson RM, et al. Effects of volume loading and pressor agents in idiopathic orthostatic tachycardia. Circulation 1997; 96: 575-80.

21. Grubb BP, Kosinski DJ, Boehm K, Kip K. The postural orthostatic tachycardia syndrome: a neurogenic variant identified during head-up tilt table testing. Pacing Clin Electrophysiol 1997; 20 (9 Pt 1): 2205-12.

22. Grubb BP, Klingenheben T. Postural orthostatic tachycardia syndrome (POTS): etiology, diagnosis and therapy. Med Klin (Munich) 2000; 95 (8): 442-6.

23. Carothers B, Schmidt L \& Puri Y. Case reports and review of Postural Orthostatic Tachycardia syndrome (POTS). J Ky Med Assoc 2003; 101 (12): 549-52.

24. Dieh L RR. Continuous progression of orthostatic tachycardia as a further feature of the postural tachycardia syndrome. Pacing Clin Electrophysiol 2005; 28 (9): 975-9.

25. Stewart JM, Weldon A. Inappropriate early hypotension in adolescents: a form of chronic orthostatic intolerance with defective dependent vasoconstriction. Pediatr Res 2001; 50 (1): 97-103.

26. Novak V, Novak P, Opher-Gehrking TL, Low PA. Postural tachycardia syndrome: time frequency mapping. J Auton Nerv Syst 1996; 61 (3): 313-20. 